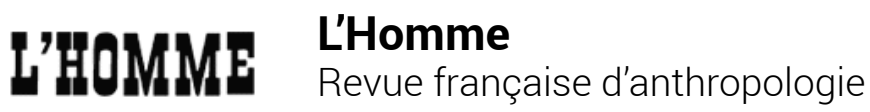

177-178 | 2006

Chanter, musiquer, écouter

\section{Judith Karafiáth \& Marie-Claire Ropars, eds, Pluralité des langues et mythe du métissage. Parcours européen}

Saint-Denis, Presses universitaires de Vincennes, 2004, 206 p., notes bibliogr.

\section{Magali Molle}

\section{(2) OpenEdition}

\section{Journals}

Édition électronique

URL : http://journals.openedition.org//homme/2320

DOI : 10.4000/lhomme.2320

ISSN : 1953-8103

Éditeur

Éditions de l'EHESS

Édition imprimée

Date de publication : 1 juin 2006

Pagination : $565-566$

ISSN : 0439-4216

Référence électronique

Magali Molle, « Judith Karafiáth \& Marie-Claire Ropars, eds, Pluralité des langues et mythe du métissage. Parcours européen », L'Homme [En ligne], 177-178 | 2006, mis en ligne le 12 avril 2006, consulté le 24 septembre 2020. URL : http://journals.openedition.org//homme/2320 ; DOI : https://doi.org/10.4000/ Ihomme.2320

Ce document a été généré automatiquement le 24 septembre 2020 .

(c) École des hautes études en sciences sociales 


\section{Judith Karafiáth \& Marie-Claire Ropars, eds, Pluralité des langues et mythe du métissage. Parcours européen}

Saint-Denis, Presses universitaires de Vincennes, 2004, 206 p., notes bibliogr.

\section{Magali Molle}

1 JUDITH Karafiáth et Marie-Claire Ropars nous présentent quelques textes issus d'un colloque organisé en France en novembre 2001, à l'occasion de la saison culturelle franco-hongroise accompagnant la naissance d'une Europe élargie. Ces articles interrogent la pluralité des langues et par là même proposent une réflexion sur la question du « métissage $»^{1}$.

2 L'ouvrage s'articule en trois parties, chacune abordant le sujet de manière particulière, mais démontrant toutes l'aptitude de chaque langue à être le double d'une autre, à devenir étrangère à elle-même à travers l'histoire ; étrangère au sujet dont elle serait la «langue maternelle» quand celui-ci est mis en contact avec d'autres langues qu'il adopte...

3 La première approche concerne les " migrations ", le " voyage des langues " à travers le temps et l'espace, retraçant ainsi l'évolution des langues du Moyen Âge à notre actuelle modernité, recontextualisant le statut de langues : la place du français (notamment lors de la politique d'assimilation en Algérie), les pérégrinations du latin, de l'allemand, de l'italien et du hongrois au cours de l'histoire et des luttes politiques.

Dans le premier article, Christopher Lucken pose la question du statut du versant maternel de la langue, langue de l'enfance, intuitive, complètement intégrée par le sujet, par rapport à son versant paternel, langue de « la raison », langue apprise, langue à laquelle on ne pourrait être qu'étranger. L'auteur interroge le sens de l'expression "langue maternelle ». Existerait-il réellement une langue " unique », la seule que l'on posséderait et à laquelle on appartiendrait, sous prétexte qu'elle serait la première, utilisée dès les premiers mots? Une «langue totale avec laquelle on entretient un lien naturel et quasi biologique, génétique, qui la rend irremplaçable et inaliénable» 
(p. 11), une langue partagée, constitutive d'identité et de communauté, à laquelle toutes les autres langues seraient étrangères, barbares? Chaque langue ne serait-elle pas plutôt plurielle, ne serait-elle pas toujours «l'union d'éléments hétérogènes qui ne cessent d'entrer en conflit»(p.12)? L'auteur retrace ainsi le contexte historique, social, politique et linguistique dans lequel apparaît cette notion de «langue maternelle».

Dans un deuxième article, Tivadar Gorilovics résume le statut du latin en Hongrie depuis un demi siècle, ce qui suscite de nombreux débats parmi les écrivains et journalistes hongrois.

6 Nous reprendrons un dernier article de cette approche qui concerne la réécriture et la réception des Mémoires de Casanova au fil de ses nombreuses traductions et remaniements ${ }^{2}$. Ilona Kovacs raconte les aventures de l'œuvre de Casanova, et explique comment un texte pourra être porteur de sens différents selon les versions qui en sont écrites. Ainsi l'Europe centrale préfère le cliché du personnage exilé, la figure du prisonnier évadé des Plombs, à celui de l'aventurier séducteur, représentation inscrite principalement dans l'esprit de l'Europe occidentale sur le modèle de Don Juan. Ces stéréotypes ont ainsi conduit à une lecture fictionnalisée du texte, plutôt qu'à une analyse historique.

7 Une seconde approche, "La langue plurielle", aborde la question du métissage à travers des cas particuliers d'écrivains ou de créations littéraires multilingues. Ainsi le multilinguisme vécu et éprouvé par Fernando Pessoa, Ivan Blatny et Arhur Koestler nous aide à comprendre la richesse mais aussi, et surtout, la difficulté de cette existence plurilingue. L'analyse de Judith Karafiáth de l'œuvre d'Arthur Koestler nous permet de percevoir comment le multilinguisme peut être ressenti comme un tourment, un "conflit des langues dans la pensée » et provoquer une incertitude identitaire. Le cas de Yvan Blatny est également très significatif à ce sujet. Ainsi nous sont montrés les paradoxes d'une existence linguistique plurielle, expérience difficile, torturante pour certains, pathologique pour d'autres, et pourtant culturellement très riche.

8 La dernière partie, "Duplicité du métissage », interroge l'inévitable duplicité des syncrétismes transculturels. En analysant la ville de Trieste et le mythe de sa plurinationalité heureuse, Anne-Rachel Hermetet démontre elle aussi la difficulté d'une expérience de mélange culturel. En effet, alors que les légendes, en France, présentent Trieste comme un monde de métissage harmonieux, lieux où cohabitent paisiblement Italiens et Slovènes, la réalité montre plutôt la division de Trieste en deux parties distinctes qui s'opposent.

9 Le dernier article concerne Ismaïl Kadaré et la tristesse du «métissage». Bruno Clément expose deux textes de Kadaré, une monographie sur Eschyle et une nouvelle, L'Église Sainte-Sophie. L'auteur conclut ainsi son article: "La tristesse, l'éternelle tristesse d'Eschyle, est celle d'un métissage manqué, peut-être impossible» (p. 206). Nous percevons de nouveau la difficulté du «métissage ", qui semble même peut-être irréalisable?

10 Cet ouvrage, pluriel, à l'image de son objet, donne un aperçu de la rencontre entre cultures européennes par le biais d'une analyse des langues. Il nous amène à comprendre le «métissage » en termes d'hétérogénéité, de pluralité, de partage et non de synthèse. L'expérience du " métissage » semble être souvent vécue de façon torturée par les hommes de lettre qui se perdent dans leur multilinguisme, mais qui y trouvent 
également toute leur richesse. Chaque culture se construit à partir de la rencontre avec l'Autre. La pluralité, le mélange interculturel qui n'efface pas mais donne à voir la différence, voilà peut-être le mot d'ordre de l'Europe de demain.

\section{NOTES}

1.. Nous plaçons des guillemets puisque les auteurs ont précisé la prudence avec laquelle ils ont utilisé ce terme tout au long de l'ouvrage.

2.. À partir de 1789, Giacomo Girolamo Casanova écrit en français l'Histoire de ma vie, œuvre plus connue sous le titre de Mémoires de Casanova. Jusqu'à sa mort il va retravailler son texte, le réécrire, le compléter, ayant pour objectif de le faire publier de son vivant. Cela ne sera pas le cas. En 1821, son fils vend le manuscrit de Casanova à un éditeur. Ce n'est que cent quarante ans plus tard que le manuscrit se verra réellement édité. Une première publication d'une traduction allemande paraîtra entre 1822 et 1828. Face à l'engouement des lecteurs qui souhaitent une traduction en français, l'éditeur propose une version révisée par Jean Laforge dès 1826. L'ouvrage est confronté à diverses censures, qui, au fil du temps, modifieront quelque peu le texte. La version originale du manuscrit ne sera publiée que bien plus tard aux éditions GarnierFlammarion, en 1977. 